\title{
The $r$-Neighborhood Graph: An Adjustable Structure for Topology Control in Wireless Ad Hoc Networks
}

\author{
Andy An-Kai Jeng and Rong-Hong Jan, Member, IEEE
}

\begin{abstract}
In wireless ad hoc networks, constructing and maintaining a topology with lower node degrees is usually intended to mitigate excessive traffic load on wireless nodes. However, keeping lower node degrees often prevents nodes from choosing better routes that consume less energy. Therefore, the trade-off is between the node degree and the energy efficiency. In this paper, an adjustable structure, named the $r$-neighborhood graph, is proposed to control the topology. This structure has the flexibility to be adjusted between the two objectives through a parameter $r, 0 \leq r \leq 1$. More explicitly, for any set of $n$ nodes, the maximum node degree and power stretch factor can be bounded from above by some decreasing and increasing functions of $r$, respectively. Specifically, the bounds can be constants in some ranges of $r$. Even more, the $r$-neighborhood graph is a general structure of both $R N G$ and $G G$, two well-known structures in topology control. Compared with $Y G_{k}$, another famous adjustable structure, our method always results in a connected planar with symmetric edges. To construct this structure, we investigate a localized algorithm, named $P L A$, which consumes less transmitting power during construction and executes efficiently in $O(n \log n)$ time.
\end{abstract}

Index Terms-Wireless ad hoc networks, topology control, energy-efficient, localized algorithm.

\section{INTRODUCTION}

W IRELESS ad hoc networks enhance the conventional deployment of communicating environments for many applications, such as conferences, hospitals, battlefields, search and rescue teams, etc. In these environments, the performance of network operations heavily depends upon the underlying topology [4]. For instance, the delivery rate would be significantly lower as the underlying topology breaks. Therefore, appropriately controlling the topology is a crucial stage in communication. The topology control problem in wireless ad hoc networks has been widely studied in recent years [3], [15], [18], [19], [20], [23], [29], [32]. Generally speaking, the core of this problem is to determine a set of wireless links such that the composed topology is able to achieve certain goals [23]. These goals would be variant depending upon the circumstances and could be either qualitative features or quantitative objectives. Since wireless nodes usually struggle with limited bandwidth and computation power, a genius way should be able to simultaneously achieve several goals. In this paper, we aim to control the topology with the following goals, which are extremely desired in wireless environments:

1. Symmetry. The existence of asymmetric links may complicate many communication primitives. For instance, the MAC layer's ACK is hard to implement

- The authors are with the Department of Computer and Information Science, National Chiao Tung University, 1001 Ta Hsueh Road, Hsinchu, 300, Taiwan, ROC. E-mail: \{andyjeng, rhjan\}@cis.nctu.edu.tw.

Manuscript received 7 July 2005; revised 24 Jan 2006; accepted 17 Mar. 2006; published online 25 Jan. 2007.

Recommended for acceptance by K. Nakano.

For information on obtaining reprints of this article, please send e-mail to: tpds@computer.org, and reference IEEECS Log Number TPDS-0323-0705.

Digital Object Identifier no. 10.1109/TPDS.2007.1004. when some links are not bidirectional [21]. Besides, asymmetric links in topology would also cause inconsistent routing qualities at two ends.

2. Connectivity. Connectivity is unquestionably the most essential prerequisite in any communicable topology [23]. Two nodes $u$ and $v$ are strongly connected if there is a directed path from $u$ to $v$ and vice versa. A directed topology is strongly connected if all pairs of nodes are strongly connected. If the links are symmetric, we should aim at the connectivity of an undirected topology instead.

3. Energy efficiency. Energy is the most crucial resource in wireless nodes. Due to the severe path loss in radio carriers, transmitting with large ranges would exponentially run out of nodes' energy. Therefore, relaying messages through multiple hops with shorter ranges could usually consume less energy [24]. How to choose the links between nodes for relaying is a critical point in this goal.

4. Sparseness. Numerous distributed and localized routing protocols are based on flooding [13]; however, this may burden networks with unavoidable redundant messages. Thus, keeping a sparse topology, consisting of linear number of links [15], would be an ingenious way to shrink the expenditure from network operations.

5. Maximum node degree. For some nodes with overly large degrees, the network flows will concentrate on them and rapidly draw out their energy. Besides, a larger node degree means tighter dependency among nodes, which is not expected when wireless nodes move frequently. Therefore, the maximum node degree over a topology should be bounded from above by some constant. 
6. Planarity. A graph is planar if it has no crossed links inside. It is helpful for many geometric problems: The shortest path (least energy unicast route) can be quickly found in linear time when the underlying topology is planar [12]. Besides, in many positionbased routing algorithms, the successful delivery can be guaranteed only if the underlying topology is planar [2], [11].

Taking a further look, a topology having constant node degree must be sparse. So, we can be concerned with the fifth goal only. Unfortunately, keeping nodes with lower node degree would possibly sacrifice some potential links composing more energy efficient routes in topology. Therefore, empirically, a trade-off is between the node degree and energy efficiency [15]. For this reason, we aim to design an adjustable way so that the trade-off can be adjusted flexibly.

In wireless ad hoc networks, due to the absence of a central arbitrator and the limited sensing range, centralized approaches [3], [30] are rarely attainable. Therefore, a variety of distributed approaches were proposed [17], [19], [29]. A distributed protocol passes messages hop-byhop. However, this may cause considerable overhead through the entire network. So, a localized approach is more preferred. According to Stojmenovic and Lin [27], a node using the localized topology control method requires information within constant hop(s). However, in some localized approaches [15], [16], [18], [27], the operations should recursively depend upon the computed status or partial results from nearby nodes, which may hurt their practicability. Therefore, in the following, we define a new type of methodology for more practicability:

Definition 1. An algorithm $L$ is purely localized if it is localized and all operations depend upon only the information inherent ${ }^{1}$ in nodes, available before any execution of $L$.

A purely localized topology control algorithm is more useful to large-scale and high mobility environments, since the operation of a node is completely isolated from any execution of other nodes. Further, we say that a structure is purely localizable if we can construct it by a purely localized algorithm. Our goal is to investigate a purely localizable structure so that all the desired goals listed above can be achieved.

The rest of this paper is organized as follows: Section 2 specifies the network model and formally describes the problem under study. In Section 3, we review and summarize the related works. The main structure, components, and their theoretical results are presented in Section 4. Some detailed derivations are given in the Appendix. In Section 5, we investigate an extended version of the main structure to comprehend our theoretical properties. In Section 6, a purely localized algorithm is investigated to construct our structure. Finally, concluding remarks and some directions for further research are given in the last section.

\section{The Model and Problem}

The wireless ad hoc network discussed in this paper consists of a set $V$ of $n$ wireless nodes distributed on a

1. The node's position and id are usually assumed to be inherited in nodes. See Section 2 for more explanation. two-dimensional plane $\Re^{2}$. Each node is equipped with an omnidirectional antenna and can change its transmission range by adjusting the transmitting power at any level. The maximum transmission ranges are equal among all nodes. In other words, we can normalize the maximum transmission ranges of all nodes to be 1 for simplicity. In addition, each node $u$ can obtain its position $P(u)$ through a lowerpower GPS or some other ways [14], and a unique $i d(u)$ is also available to each node $u$.

This network can be modeled as a unit disk graph, $U D G(V)$. In this graph, an edge uv exists if and only if the euclidean distance between $u$ and $v$, denoted as $\|u v\|$, is at most 1.

The least power required to transmit immediately between $u$ and $v$ is modeled as $\|u v\|^{\alpha}$, where $\alpha$ is typically taken on a value between 2 and 4, depending on the attenuation strength of the communication environment [5]. To measure the power efficiency of a topology, Li et al. [15] defined a well-formed measure, named power stretch factor. We reintroduce it below. Let $\pi(u, v)=v_{0} v_{1} \ldots v_{h-1} v_{h}$ be a unicast path connecting nodes $u$ and $v$, where $v_{0}=u$ and $v_{h}=v$. The total transmission power consumed by path $\pi(u, v)$ is defined as

$$
p(\pi(u, v))=\sum_{i=1}^{h}\left\|v_{i-1} v_{i}\right\|^{\alpha} .
$$

Let $\pi_{G(V)}^{*}(u, v)$ be the least-energy path connecting $u$ and $v$ in graph $G(V)$. Given a subgraph $G^{\prime}(V)$ in $U D G(V)$, the power stretch factor of $G^{\prime}(V)$ with respect to $U D G(V)$ is defined as

$$
\rho(G(V))=\max _{u, v \in V} \frac{p\left(\pi_{G^{\prime}(V)}^{*}(u, v)\right)}{p\left(\pi_{U D G(V)}^{*}(u, v)\right)} .
$$

On the other hand, the maximum node degree of graph $G(V)$ is defined as

$$
d_{\max }(G(V))=\max _{u \in V} d_{G(V)}(u),
$$

where $d_{G(V)}(u)$ is the degree of node $u$ in graph $G(V)$.

\section{Related Work}

Many localizable structures, used to control the network topology, have been proposed in the literature [15], [16], [18], [26], while only a few of them are purely localizable. In the following, we list four well-known structures. Most of them or their extensions are purely localizable:

- The constrained Relative Neighborhood Graph [28], denoted by $R N G(V)$, has an edge $u v$ if and only if $\|u v\| \leq 1$ and the intersection of two open disks ${ }^{2}$ centered at $u, v$ with radius $\|u v\|$ contains no node $w \in V$; see Fig. 1a.

- The constrained Gabriel Graph [6], denoted by $G G(V)$, has an edge $u v$ if and only if $\|u v\| \leq 1$ and the open disk using $\|u v\|$ as diameter contains no node $w \in V$; see Fig. $1 b$.

2. An open disk centered at point $x$ with radius $d$ is the collection of points with distance less than $d$ from $P(x)$. 


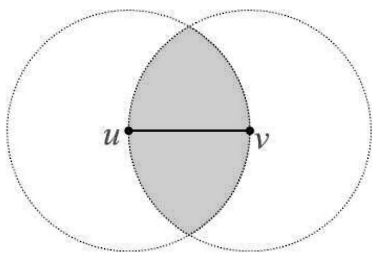

(a)

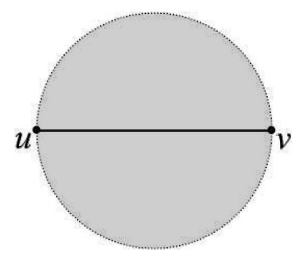

(b)

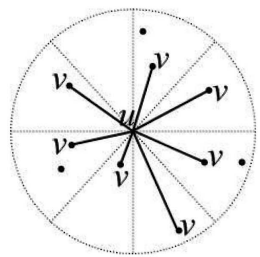

(c)

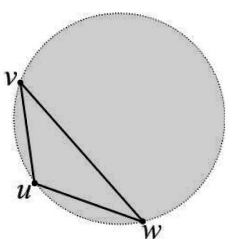

(d)

Fig. 1. (a) $R N G(V)$. (b) $G G(V)$. (c) $Y G_{k}(V), k=8$. (d) $U \operatorname{Del}(V)$.

- The constrained Yao Graph [33] with a parameter $k \geq 6$, denoted by $\overrightarrow{Y G}_{k}(V)$, is constructed as follows: For each node $u$, define $k$ equal cones by $k$ equalseparated rays originated at $u$. At each cone, a directed edge $u v$ exists if $\|u v\| \leq 1$ and the cone contains no vertex $w \in V$ such that $\|u w\|<\|u v\|$. Ties are broken arbitrarily. $Y G_{k}(V)$ is denoted as the underlying undirected graph of $\overrightarrow{Y G}_{k}(V)$; see Fig. 1c.

- A Delaunay Triangulation, denoted by $\operatorname{Del}(V)$, is a triangulation of $V$ in which the interior of the circumcircle of each $\Delta u v w$ contains no node $w \in V$. The unit Delaunay Triangulation, denoted by $U \operatorname{Del}(V)$, has all edges of $\operatorname{Del}(V)$ except those longer than 1 [8], [18]; see Fig. 1d.

Let us discuss the properties of these structures and their extensions. We say an objective $f($.) of a structure $S(V)$ is bounded if there is a constant $C$ such that $f(S(V)) \leq C$, for any set $V$ of $n$ nodes. Li et al. [15] showed that $d_{\max }(R N G(V))$ is unbounded if there is a node $u \in V$ having an unbounded number of neighbors adjacent to $u$ at exactly the same distance in the underlying $U D G(V)$. To overcome this problem, Wattenhofer and Zollinger [32] proposed an algorithm to find a structure, denoted by $X T C(V)$. They showed that $X T C(V)$ is a subgraph of $R N G(V)$ and the $d_{\max }(X T C(V))$ is at most 6 . Especially, if there is no node having two or more neighbors at exactly the same distance in $V, X T C(V)$ is identical to $R N G(V)$ [24]. Their results infer the following theorem:

Theorem 1. Given a set $V$ of nodes on $\Re^{2}$, if there is no node having two or more neighbors at exactly the same distance, then $d_{\max }(R N G(V)) \leq 6$.

We denote the condition in Theorem 1 as Assumption $A S$. That is,

Assumption AS. There is no node in $V$ having two or more neighbors at exactly the same distance.

This theorem reveals that even $R N G(V)$ has no constant bound on its node degree. It is still useful since the distances of nodes in the real world are rarely exactly the same. The constrained Gabriel Graph $G G(V)$ has the least power stretch factor 1 in comparison with the unbounded power stretch factor $n-1$ of $R N G(V)$ [15]. However, $d_{\max }(G G(V))$ could be as large as $n-1$. An extended structure, Enclosure graph [16], [14], [24], denoted by $E G(V)$, is generalized from $G G(V)$. It can always result in a subgraph of $G G(V)$ [16]. Even so, its maximum node degree is still unbounded [20], [24].
To overcome the trade-off between the maximum node degree and the power stretch factor, an adjustable structure, having the flexibility to be adjusted between the two objectives, becomes more attractive. $\overrightarrow{Y G}_{k}(V)$ is an adjustable structure. It can be adjusted through a parameter $k$ such that, for any given $k$, the maximum out-degree is at most $k$, and the power stretch factor is at most $1 /\left(1-(2 \sin \pi / k)^{\alpha}\right)$ [15]. We say an objective $f($.$) of an adjustable structure S_{k}(V)$ with parameter $k$ is partially bounded if there is at least one $k_{0}$ such that $f\left(S_{k_{0}}(V)\right)$ is bounded. According this definition, the maximum out-degree and power stretch factor of $\overrightarrow{Y G}_{k}(V)$ are partially bounded since, for some ranges of $k, k$ and $1 /\left(1-(2 \sin \pi / k)^{\alpha}\right)$ are constants. However, the asymmetric edges of $\overrightarrow{Y G}_{k}(V)$ may lead to large in-degrees even when $k$ is very small [15]. So, $d_{\max }\left(Y G_{k}(V)\right)$ can be neither bounded nor partially bounded. To improve this, an extension of $\overrightarrow{Y G}_{k}(V)$, named Yao and Sink, was proposed [15], [17], [29]. It can limit the maximum node degree in $(k+1)^{2}-1$ and result in symmetric edges. Unfortunately, in this structure, the neighbors of some node should be recursively determined by one another so that it cannot be purely localizable. The unit Delaunay triangulation $U \operatorname{Del}(V)$ has a bounded power stretch factor. However, neither $\operatorname{Del}(V)$ nor $U \operatorname{Del}(V)$ can be computed locally. So, Li et al. [18] suggested a localized version of the Delaunay graph, denoted by $L D e l^{(h)}(V)$, where $h$ means that each node uses at most $k$-hop information. The power stretch factor of $L D e l^{(k)}(V)$ is bounded for all $k \geq 1$. Even so, its maximum node degree is not bounded for any $h$.

The relations among these structures were studied in several papers [7], [10], [16], [22], [24], [33]. We summarize them in Fig. 2, where $\operatorname{EMST}(V)$ is the euclidean minimum spanning tree of $U D G(V)$. With these relations, their connectivity and planarity can be easily inferred.

Regarding their connectivity, we know that $\operatorname{EMST}(V)$ is connected if $U D G(V)$ is itself a connected component of $V$. Therefore, when $U D G(V)$ is connected, all graphs containing $\operatorname{EMST}(V)$ are connected. That is, $R N G(V), G G(V), E G(V)$, $U \operatorname{Del}(V), \operatorname{LDel}^{(k)}(V)$, and $Y G_{k}(V)$ are all connected. The connectivity of $X T C(X)$ was proven in a different way [24].

Regarding their planarity, $L D e l^{(k)}(V)$ is planar for any $k \geq 2$ [18]. Therefore, all subgraphs of $\operatorname{LDel}^{(2)}(V)$ are planar. That is, $\operatorname{UDel}(V), G G(V), E G(V), R N G(V), X T C(V)$, and $\operatorname{EMST}(V)$ are all planar. On the contrary, $\overrightarrow{Y G}_{k}(V)$ and $L D e^{(1)} l(V)$ cannot avoid producing a crossed link, so they are not planar [15], [18]. Table 1 summarizes the above discussion. 


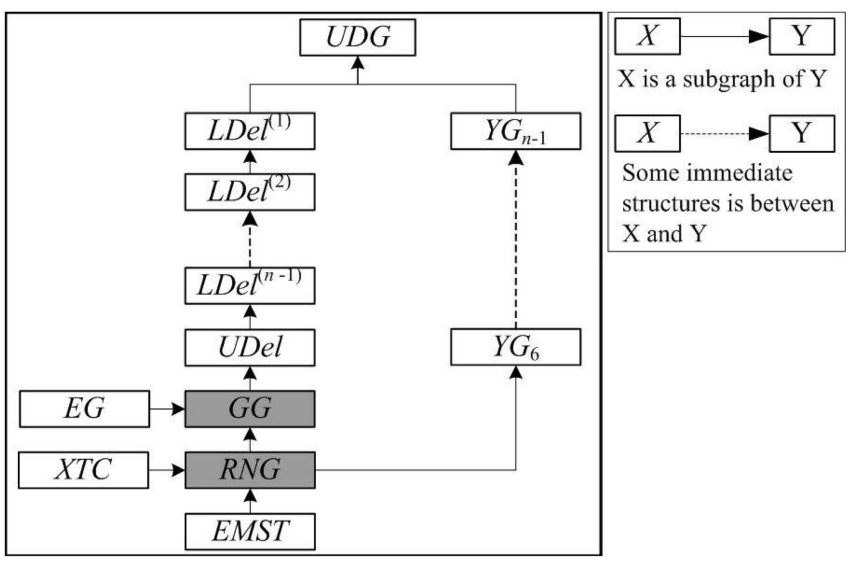

Fig. 2. The relations of the purely localizable structures and their extensions.

From Table 1, we can see that no presented structure can bound or even partially bound the two objectives. Besides, to the best of our knowledge, no other structure can be purely localizable and achieve this goal. Therefore, we will propose the first purely localizable structure, named $r$-Neighborhood Graph, to fill this gap. This structure is adjustable and can always result in a connected planar with symmetric edges. In addition, we can show that our structure is a generation of both $G G(V)$ and $R N G(V)$.

Apart from the purely localizable structures, several composite methods, based on combining two or more existent structures, were investigated in the last few years [17], [19], [25], [31]. Conceptually, the main idea is to use the virtue of one structure to patch up the fault in the other structures. For examples, the ordered Yao structure, denoted as $\operatorname{OrdYao}(V)$ [1], is a variation of $Y G_{k}^{*}(V)$. It has the partially bounded maximum node degree and length stretch factor. However, the planarity cannot be guaranteed. Therefore, Li and Wang [19], [31] applied $\operatorname{OrdYao}(V)$ onto $\mathrm{LDel}^{(2)}(V)$ to avoid the crossed edges produced by $\operatorname{OrdYao}(V)$; Song et al. [25] improved it by applying the $\operatorname{OrdYao}(V)$ on $G G(V)$ using only one-hop information. However, the construction of $\operatorname{OrdYao}(Y)$ requires exchanging the computed status as well as partial results between nodes. Consequently, none of them is purely localized or purely localizable.

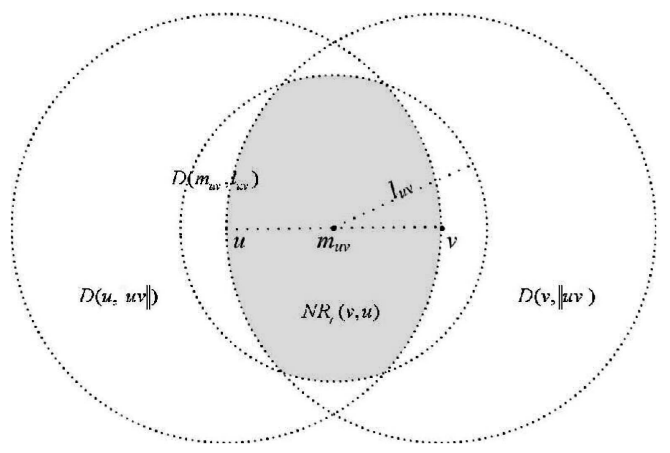

Fig. 3. The $r$-neighborhood region of nodes $u$ and $v$.

\section{The $\boldsymbol{r}$-NeighboRHOOd GRAPH}

In this section, we introduce a new adjustable structure. First, we define a region on $\Re^{2}$. It will be used to compose our structure. Let $x$ be any point on $\Re^{2}$ and the open disk and circle centered at $P(x)$ with radius $d$ are denoted as $D(x, d)$ and $C(x, d)$, respectively. The region is defined as follows:

Definition 2. Given a node pair $(u, v)$ on $\Re^{2}$, the $r$-neighborhood region of $(u, v)$, denoted as $N R_{r}(u, v)$, is defined as

$$
N R_{r}(u, v)=D(u,\|u v\|) \cap D(v,\|u v\|) \cap D\left(m_{u v}, l_{u v}\right),
$$

where $m_{u v}$ is the middle point on $u v$,

$$
l_{u v}=(\|u v\| / 2)\left(1+2 r^{2}\right)^{1 / 2},
$$

and $0 \leq r \leq 1$.

When not confused, we use $m$ and $l$ instead of $m_{u v}$ and $l_{u v}$, respectively. In Fig. 3, the shaded region intersected by the three open disks sketches an example of the $r$-neighborhood region. This region is obviously equivalent to the following point set:

$N R_{r}(u, v)=\left\{P(x) \in \mathcal{R}^{2}\|u x\|<\|u v\|,\|v x\|<\|u v\|,\|m x\|<l\right\}$.

For any node $w$ located on $N R_{r}(u, v)$, this region limits the power consumed by path $u w v$. This property is shown in Lemma 2 and derived in the Appendix.

TABLE 1

The Properties of the Four Main Purely Localizable Structures

\begin{tabular}{|c|c|c|c|c|c|}
\hline & Power stretch factor & Maximum node degree & Planar & Symmetric & Connected \\
\hline$R N G(V)$ & Unbounded & $\begin{array}{l}\text { Bounded (with } A S) \\
\text { Unbounded (without } A S)\end{array}$ & Yes & Yes & Yes \\
\hline$G G(V)$ & Bounded & Unbounded & Yes & Yes & Yes \\
\hline$Y G_{k}(V)$ & Partially bounded & Unbounded & No & No & Yes \\
\hline$L D l^{(k)}(V)$ & Partially bounded & Unbounded & $\begin{array}{r}\text { No }(k=1) \\
\text { Yes }(k \geq 2)\end{array}$ & Yes & Yes \\
\hline
\end{tabular}


Lemma 2. Given two nodes $u$ and $v$ on $\Re^{2}$, for any node $w$ such that $P(w) \in N R_{r}(u, v), p(u w v)<\|u v\|^{\alpha}\left(1+r^{\alpha}\right)$, for all $\alpha \geq 2$.

This lemma explains why we call such a plane a neighborhood region: For any node $w$ located in the region $N R_{r}(u, v)$, it should be an alternative neighbor for $u$ with respect to $v$ in the sense that the power required for relaying from $u$ to $v$ through $w$ is no greater than $1+r^{\alpha}$ times the immediate transmission. Based on this region, the structure is defined below:

Definition 3. Given a set $V$ of nodes on $\Re^{2}$, the $r$-neighborhood graph of $V$, denoted as $N G_{r}(V)$, has an edge uv if and only if $\|u v\| \leq 1$ and $N R_{r}(u, v)$ contains no node $w \in V$, where $0 \leq r \leq 1$.

By Definition 3, if edge $u v$ is not in $U D G(V)$ or a node $w$ is inside $N R_{r}(u, v)$, there is no direct link connecting $u$ and $v$ in $N G_{r}(V)$, which means that all transmissions between $u$ and $v$ should be relayed through some other node(s) in $N G_{r}(V)$. Now, we explore the desired properties in our structure. Before this, we shall discussion the following relations:

Lemma 3. For any set $V$ of nodes on $\Re^{2}$,

$$
R N G(V) \subseteq N G_{r}(V) \subseteq G G(V),
$$

for all $0 \leq r \leq 1$.

Proof. Consider the open disk $D(m,\|u v\| / 2)$ defining $G G(V)$. Suppose $u v \in N G_{r}(V)$, the region $N R_{r}(u, v)$, has no node inside. Since $D(m,\|u v\| / 2)$ is obviously a subregion of $N R_{r}(u, v)$, for any $0 \leq r \leq 1$, there is also no node in $D(m,\|u v\| / 2)$. Therefore, according to the definition of $G G(V)$, we get $u v \in G G(V)$. On the other hand, consider the two open disks $D(u,\|u v\|)$ and $D(v,\|u v\|)$ defining $R N G(V)$. Suppose $u v \in R N G(V)$, no node is inside the intersection of $D(u,\|u v\|)$ and $D(v,\|u v\|)$, which obviously covers the region $N R_{r}(u, v)$, for any $0 \leq r \leq 1$. Therefore, no node can be inside $N R_{r}(u, v)$ and we get $u v \in N G_{r}(V)$.

Specifically, as $r=0, N R_{0}(u, v) \equiv D(m,\|u v\| / 2)$, which is the disk defining $G G(V)$. On the contrary, as $r=1$, $N R_{1}(u, v) \equiv D(m,\|u v\|)$, which is the disk defining $R N G(V)$. Therefore, $G G(V) \equiv N G_{0}(V)$ and $R N G(V) \equiv$ $N G_{1}(V)$. So, we can conclude the following theorem:

Theorem 2. The r-neighborhood graph is a generalized structure of both the restricted Gabriel graph and the restricted relative neighborhood graph.

Since a subgraph of a planar graph is always planar, and a supergraph of a connected graph is always connected, with the planarity of $G G(V)$ and connectivity of $R N G(V)$, we can infer the following two theorems:

Theorem 3. For any set $V$ of nodes on $\Re^{2}, N R_{r}(V)$ is planar, for all $0 \leq r \leq 1$.

Theorem 4. For any set $V$ of nodes on $\Re^{2}$, if the underlying $U D G(V)$ is connected, $N R_{r}(V)$ is connected, for all $0 \leq r \leq 1$.
Now, we consider the energy efficiency and node degree of $N R_{r}(V)$. We will show that the upper bound of $\rho\left(N G_{r}(V)\right)$ is increased by $r$ and, contrarily, the upper bound of $d_{\max }\left(N G_{r}(V)\right)$ is decreased by $r$. In other words, the $r$-neighborhood graph is adjustable to the two objectives through the parameter $r$. With these results, we can further show that the power stretch factor and maximum node degree are partially bounded in our structure. Before these, a property proposed by Li et al. [15] shall be mentioned. It can be used to simplify our proof.

Lemma 4 [15]. Given a subgraph $G^{\prime}(V) \subseteq U D G(V)$ and a constant $C, \rho\left(G^{\prime}(V)\right) \leq C$ if and only if, for any edge uv in $G(V)$, there is a path $\pi(u, v)$ in $G^{\prime}(V)$ such that $p_{G^{\prime}(V)}(u, v) \leq C\|u v\|^{\alpha}$.

This lemma indicates that to derive an upper bound for $\rho\left(N G_{r}(V)\right)$, it is sufficient to consider only those node pairs having direct links in $U D G(V)$. So, we aim to derive a strictly decreasing function $F(r)$, such that, for any $u v$ in $U D G(V)$, a path $\pi(u, v)$ is in $N R_{r}(V)$ such that $p(\pi(u, v)) \leq F(r)\|u v\|^{\alpha}$. To achieve this, we investigate an algorithm called EXPANSION with an input of any two nodes $(u, v)$ that outputs subgraph $S$ of $N R_{r}(V)$ related to $(u, v)$. Let $P(S)$ be the total transmission power of edges in $S$, i.e., $P(S)=\sum_{s t \in S} p(s, t)$. We can show that there is some path in $S$ connecting $(u, v)$ and $P(S) \leq F(r)\|u v\|^{\alpha}$.

\section{ALGORITHM EXPANSION}

Input: A node pair $(u, v)$ in $V$.

Output: A subgraph $S$ and a positive value $P$.

Step 1: $S=\{\}, S^{\prime}=\{(u, v)\}, Q=\{u, v\}, P=\|u v\|^{\alpha}$;

Step 2: When some node pair $(s, t)$ is in $S$ such that a node $w \in N R_{r}(s, t)$

$$
\begin{aligned}
& S^{\prime}=S^{\prime}-(s, t) ; \\
& \text { If } w \notin Q \text { then } \\
& \quad \begin{array}{l}
S^{\prime}=S^{\prime} \cup(s, w) \cup(w, t) ; \\
Q=Q \cup\{w\} ; \\
P=P+(\|s t\| r)^{\alpha} ;
\end{array}
\end{aligned}
$$

Otherwise,

$$
S^{\prime}=S^{\prime} \cup(s, w) ;
$$

Step 3: $S=\left\{x y \in N G_{r}(V) \mid(x, y) \in S^{\prime}\right\}$;

Step 4: Stop and output $E$ and $P$.

In this algorithm, $S^{\prime}$ is a set of node pairs in which an edge st in $N R_{r}(V)$ can be a part of $S$ only if its two ends $(s, t)$ are in $S^{\prime}$ as described at Step 3. So, to determine $S$, we have to discuss the $S^{\prime}$ first. Initially, $S^{\prime}$ contains only $(u, v)$. Then, it will be recursively expanded as follows: For each $(s, t)$ in $S^{\prime}$, if a node $w$ is in $N R_{r}(s, t)$ and not considered before, replace $(s, t)$ with $(s, w)$ and $(w, t)$; if a node $w$ is in $N R_{r}(s, t)$ but considered before, replace $(s, t)$ with $(s, w)$; otherwise, keep $(s, t)$ unchanged. We use the set $Q$ to record the considered nodes.

When some $(s, t)$ is in $S^{\prime}$ such that a node $w \in N R_{r}(s, t)$, no matter whether $w$ is considered or not, by (1), the replaced node pair(s) must be shorter than $\|s t\|$, i.e., $\|s w\|<$ $\|s t\|$ and $\|w t\|<\|s t\|$. Thus, after finite iterations, each node pair in $S^{\prime}$ can be replaced by another node pair with the shortest distance. So, the algorithm is terminable. Now, we show that $(u, v)$ is connected by some path in the subgraph $S$ when termination occurs. 
Lemma 5. Given any set $V$ of node on $\Re^{2}$, for any two nodes $u$ and $v$ in $V$, if edge $u v$ is in $U D G(V)$ and $U D G(V)$ is connected, there is some path in $S$ connecting $(u, v)$.

Proof. Since $Q$ includes $u$ and $v$, we can prove this lemma by showing that all nodes in $Q$ are connected in $S$. For each expansion of $S^{\prime}$, we define a dummy graph $S^{\prime \prime}$ in which an edge st exists if and only if $(s, t)$ is in $S^{\prime}$ (note that any edge in $S^{\prime \prime}$ is not necessarily in either $U D G(V)$ or $N R_{r}(V)$ ). First, we show that, at any iteration, all considered nodes in $Q$ are connected by $S^{\prime \prime}$. Initially, $Q$ is connected by $S^{\prime \prime}$, since $S^{\prime}=\{(u, v)\}$ and $Q=\{u, v\}$. We assume for induction that all nodes in $Q$ are connected by $S^{\prime \prime}$ at the $k$ th iteration. Then, we show that it is true for the next iteration. At the $k+1$ th iteration, if there is no pair in $S^{\prime}$ that satisfies the entrance condition of Step 2, the claim is correct, since $Q$ and $S^{\prime \prime}$ are unchanged; otherwise, a node pair $(s, t) \in S^{\prime}$ is expended. In this case, if the chosen $w \notin Q, w$ is connected with all nodes in $Q$ via dummy edges $s w$ and $w t$; otherwise, $w \in Q$, which implies that all nodes in $Q$ are still connected by $S^{\prime \prime}$ as in the previous iteration. As described above, the distance of any expended node pair is no longer than the previous one. So, if $u v$ is in $U D G(V)$, all edges in $S^{\prime \prime}$ are also in $U D G(V)$. Then, as the algorithm proceeds to Step 3, no nodes can be in the $r$-neighborhood region of any node pair in $S^{\prime}$. With these two facts, all dummy edges in $S^{\prime \prime}$ are also in $N R_{r}(V)$ at termination. So, $S$ is equivalent to the last $S^{\prime \prime}$. Consequently, if $U D G(V)$ is connected, by Theorem 4, all nodes in the last $Q$ are connected to $S$. $\square$ Then, we derive a strictly decreasing function $F(r)$ using the value $P$ in this algorithm.

Lemma 6. Given any set $V$ of $n$ nodes on $\Re^{2}$, for any two nodes $u$ and $v$ in $V$,

$$
P(S) \leq F(r)\|u v\|^{\alpha} \text { and } F(r)=1+(n-2) r^{\alpha},
$$

for all $0 \leq r \leq 1$ and $\alpha \geq 2$.

Proof. Let $P\left(S^{\prime}\right)=\sum_{(s, t) \in S^{\prime}} p(s, t)$. We show that $P\left(S^{\prime}\right) \leq P$ at each iteration of Step 2. Initially, $S^{\prime}=\{(u, v)\}$. We can get $P\left(S^{\prime}\right)=\|u v\|^{\alpha}=P$. Then, at the first iteration, if no node $w$ is in $N R_{r}(u, v)$, the claim remains true since neither $P$ nor $S$ is changed; otherwise, a node $w$ is in $N R_{r}(u, v)$. Since there is no node except $u$ and $v$ in $Q$ so far, $(u, v)$ is replaced by $(v, w)$ and $(w, v)$. By Lemma 2,

$$
P(v w)+P(w u) \leq P(u v)\left(1+r^{\alpha}\right)=P+(\|u v\| r)^{\alpha} .
$$

Consequently, the new $P$ remains an upper bound of $P\left(S^{\prime}\right)$. We assume for induction that $P\left(S^{\prime}\right) \leq P$ at the $k$ th iteration. Then, we prove that the claim is true at the next iteration. If the entrance condition of Step 2 is not satisfied or the chosen $w \notin Q$, it can be proved by the same reasons as in the first iteration. Otherwise, $(s, t)$ is replaced by $(s, w)$ only. By (1), $P(s t) \leq P(s w)$, which implies that the unchanged $P$ is still an upper bound of $P\left(S^{\prime}\right)$. Besides, (1) further implies that the distance between any two nodes in $S^{\prime}$ is no greater than $\|u v\|$. So, another upper bound $P^{\prime}$ can be found by replacing $P=P+(\|s t\| r)^{\alpha}$ by $P^{\prime}=P^{\prime}+(\|u v\| r)^{\alpha}$. Moreover, we can observe that the situation where $w$ is chosen from some $N G_{r}(s, t)$ that is not in $Q$ never happens more than $n-2$ times, since, in this case, the size of $Q$ must be increased by 1 . Consequently, $P\left(S^{\prime}\right) \leq P \leq P^{\prime} \leq P(u v)+P(u v) r^{\alpha}(n-2)$. Finally, we get $F(r)=\left(1+r^{\alpha}\right)(n-2)$.

With Lemmas 4, 5, and 6, we can conclude the following theorem:

Theorem 5. For any set $V$ of $n$ nodes on $\Re$, for all $0 \leq r \leq 1$ and $\alpha \geq 2$,

$$
\rho\left(N G_{r}(V)\right) \leq 1+r^{\alpha}(n-2)=F(r)
$$

Although this bound is related to the node size $n$ so that $\rho\left(N R_{r}(V)\right)$ cannot be bounded, it can still be constant when $r$ is 0 or sufficiently small, i.e., $\rho\left(N R_{r}(V)\right)$ is bounded in some range of $r$. So, we can make the following conclusion:

Corollary 1. The power stretch factor of the r-neighborhood graph is partially bounded.

Consider the maximum node degree of the $r$-neighborhood graph. Since $N R_{r}(V)$ consists of all edges in $R N G(V)$, the maximum node degree of $N R_{r}(V)$ is no less than that of $R N G(V)$. In Section 3, we know that $d_{\max }(R N G(V))$ is not always bounded in any case of $V$. Thus, $d_{\max }\left(N G_{r}(V)\right)$ is also unbounded. Fortunately, Theorem 1 indicates that $d_{\text {max }}(R N G(V))$ is bounded in most cases of $V$, where $A S$ is assumed. Therefore, in the following theorem, we analyze the maximum node degree of the $r$-neighborhood graph under assumption $A S$.

Theorem 6. For any set $V$ of nodes on $\Re^{2}$ with assumption $A S$, for all $0 \leq r \leq 1$,

$$
d_{\max }\left(N G_{r}(V)\right) \leq\left|\pi / \sin ^{-1}(r / 2)\right| .
$$

Proof. To prove this statement, it is sufficient to show that, in $N G_{r}(V)$, there are no adjacent edges enclosing an angle less than $2 \sin ^{-1}(r / 2)$. Assume for contradiction that two edges $u v$ and $u w$ in $N G_{r}(V)$ enclose an angle $\theta<2 \sin ^{-1}(r / 2)$ at node $u$, where $w, v \in V$. Without loss of generality, we assume that $\|u w\|<\|u v\|$. With assumption $A S$, all nodes are placed on different positions, i.e., $P(x) \neq P(y)$, for any two nodes $x, y \in V$.

Consider the length of $v w$ : If $\angle u w v$ is obtuse, it is clear that $\|v w\|<\|u v\|$ (note that $\|v w\|$ cannot be equal to $\|u v\|$, since $P(u) \neq P(w)$ ); see Fig. $4 \mathrm{~b}$. Otherwise, if $\angle u w v$ is not obtuse, $\|v w\|$ is less $\left\|v w^{\prime}\right\|$, where $\left\|u w^{\prime}\right\|=\|u v\|$; see Fig. 4a. By the law of cosines, we have

$$
\begin{aligned}
& \left\|v w^{\prime}\right\|^{2}=\left\|u w^{\prime}\right\|^{2}+\|u v\|^{2}-2\left\|u w^{\prime}\right\|\|u v\| \cos \theta \\
& =2\|u v\|^{2}-2\|u v\|^{2} \cos \theta \\
& <2\|u v\|^{2}-2\|u v\|^{2} \cos \left(2 \sin ^{-1}(r / 2)\right) .
\end{aligned}
$$

If $\theta^{\prime}=2 \sin ^{-1}(r / 2)$, we get $\sin \left(\theta^{\prime} / 2\right)=r / 2$. Then, one of the corresponding right-angled triangles is as shown in Fig. $4 c$. In this case, $\cos \theta^{\prime}=\left(2-r^{2}\right) / 2$. Thus, we can get that $2 \sin ^{-1}(r / 2)=\theta^{\prime}=\cos ^{-}\left(\left(2-r^{2}\right) / 2\right)$. Consequently,

Equation $(2)=2\|u v\|^{2}-2\|u v\|^{2} \cos \left(\cos ^{-}\left(\left(2-r^{2}\right) / 2\right)\right)$ $=2\|u v\|^{2}-2\|u v\|^{2}\left(\left(2-r^{2}\right) / 2\right)=\|u v\|^{2} r^{2}$. 


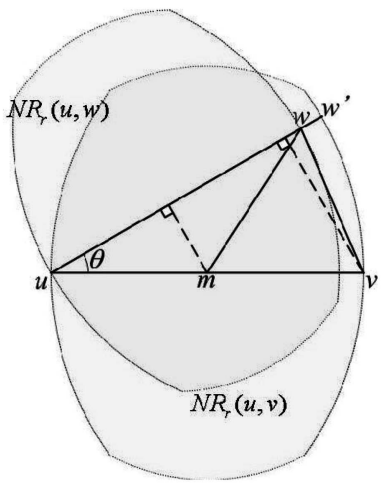

(a)

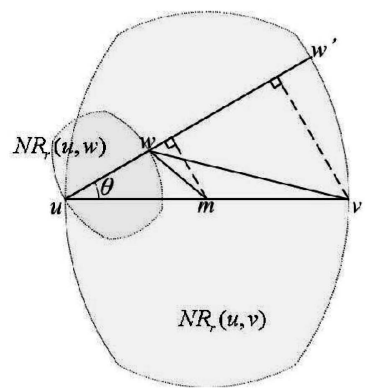

(b)

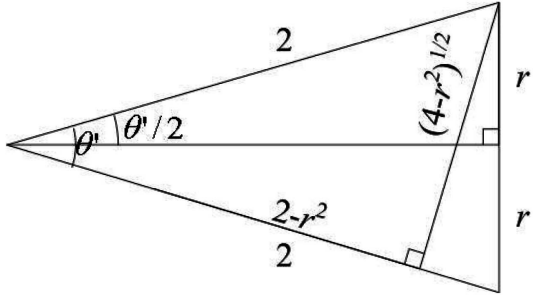

(c)

Fig. 4. (a) $\angle u w v$ and $\angle u w m$ are not obtuse. (b) $\angle u w v$ and $\angle u w m$ are obtuse. (c) A right-angled triangle with angle $\theta=2 \sin ^{-}(r / 2)$.

Consequently, we have that, for any case of $\angle u w v$,

$$
\|v w\|<\max \{\|u v\| r,\|u v\|\}=\|u v\| .
$$

Consider the length of um: If $\angle u w m$ is obtuse, $\|w m\|<\|u v\| / 2$; see Fig. 4b. Otherwise, $\|m w\|$ is less $\left\|m w^{\prime}\right\|$; see Fig. $4 \mathrm{~b}$. By the law of cosine, we have

$$
\begin{aligned}
& \left\|m w^{\prime}\right\|^{2}=\left\|u w^{\prime}\right\|^{2}+\left\|u m^{\prime}\right\|^{2}-2\left\|u w^{\prime}\right\|\left\|u m^{\prime}\right\| \cos \theta \\
& <\|u v\|^{2}+\|u v\|^{2} / 4-\|u v\|^{2} \cos \theta \\
& <5\|u v\|^{2} / 4-\|u v\|^{2}\left(\left(2-r^{2}\right) / 2\right)=\|u v\|^{2}\left(\left(1+2 r^{2}\right) / 4\right) .
\end{aligned}
$$

Similarly, we have, for any case of $\angle u w m$,

$$
\|m w\|<\max \left\{\|u v\| \sqrt{1+2 r^{2}} / 2,\|u v\| / 2\right\}=l .
$$

By (4), (6), and the assumption of $\|u w\|<\|u v\|, w$ is included in the set of points specified in (1). Therefore, $P(w) \in N R_{r}(u, v)$. However, it contradicts the assumption that $u v$ is in $N R_{r}(V)$. Thus, we conclude this theorem.

However, for those instances of $V$ without $A S$, Theorem 6 cannot hold anymore. See the instance in Fig. 5, where all nodes except $v_{i}$ are placed on the outlier of $N R_{r}\left(v_{i}, v_{1}\right)$. This will result $n-1$ neighbors adjacent to $v_{i}$ in $N R_{r}(V)$.

So, in the next section, we propose an extended version of the $r$-neighborhood graph. As the readers will see, the extended structure has the partially bounded maximum node degree for all cases of $V$ and inherits almost all of the desired features in $N R_{r}(V)$.

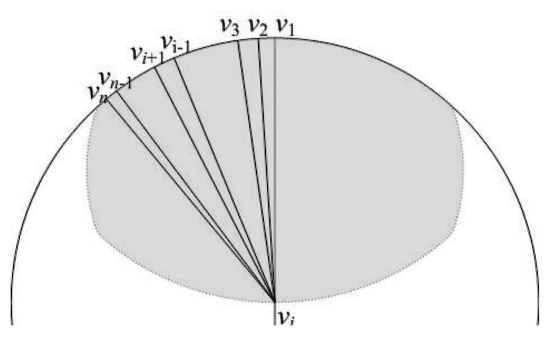

Fig. 5. $d_{\max }\left(N G_{r}(V)\right)$ is not bounded if Assumption $A S$ does not hold.

\section{The Extended $r$-NeighborhoOd GRAPH}

In this section, an extended structure of the $r$-neighborhood graph is given. The main goal is to avoid the unbounded maximum node degree in $N R_{r}(V)$. In this extension, Assumption $A S$ is not required anymore. Instead, a unique identifier $i d(u)$ is available to each node $u$ in $V$. The structure is defined as follows:

Definition 4. Given a set $V$ of nodes $\Re^{2}$, the extended $r$-neighborhood graph of $V$, denoted as $N G_{r}^{*}(V)$, has an edge $u v$ if and only if $\|u v\| \leq 1$ and there exists no node $w \in V$ satisfying one of the following three conditions:

- $\quad \mathrm{D}_{1}: P(w) \in N R_{r}(u, v)$;

- $\mathrm{D}_{2}: P(w) \in D\left(m_{u v}, l_{u v}\right) \cap C(v,\|u v\|)$ and $i d(u)>i d(w)$;

- $\mathrm{D}_{3}: P(w) \in D\left(m_{u v}, l_{u v}\right) \cap C(u,\|u v\|)$ and $i d(v)>i d(w)$.

Without $\mathrm{D}_{2}$ and $\mathrm{D}_{3}, N G_{r}^{*}(V)$ is clearly equivalent to the original r-neighborhood graph. In conditions $\mathrm{D}_{2}$ and $\mathrm{D}_{3}$, the two subregions of $D\left(m_{u v}, l_{u v}\right)$ intersected by $C(v,\|u v\|)$ and $C(u,\|u v\|)$ are, as depicted in Fig. 6, the solid left arc and the right arc along the outlier of $N R_{r}(u, v)$, respectively. When a node $w$ is located in these two arcs, the existence of edge uv should be further determined by their identifiers.

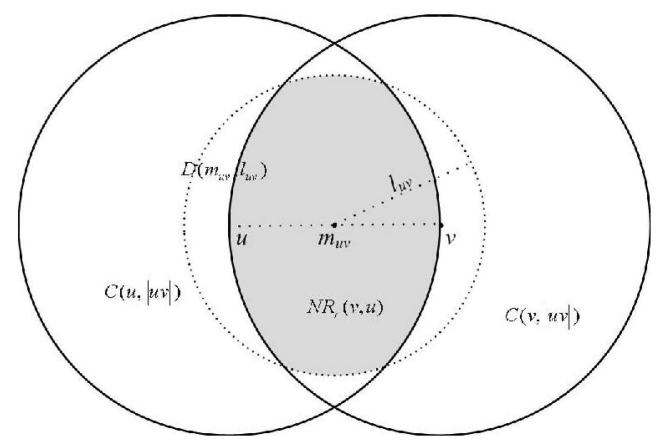

Fig. 6. The $r$-neighbor region of nodes $u$ and $v$ and the two intersections defined in $\mathrm{D}_{2}$ and $\mathrm{D}_{3}$. 


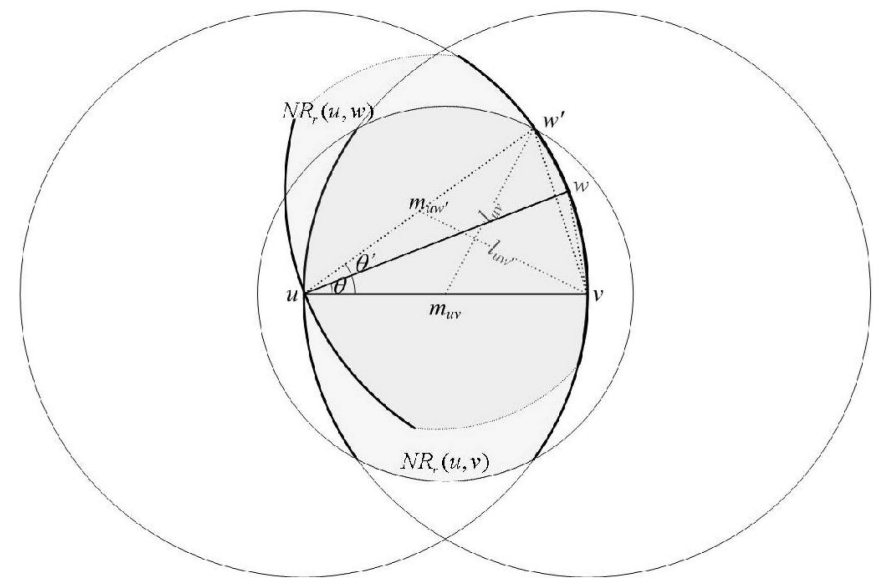

Fig. 7. If $\theta<2 \sin ^{-1}(r / 2)$ and $\|u w\|=\|u v\|$, either $u w$ or $u v$ cannot be in $N G_{r}^{*}(V)$.

Hereafter, we say that a node $w \in V$ blocks an edge $u v$ in $U D G(V)$ if and only if $w$ satisfies one of the three conditions in Definition 4.

In $N G_{r}^{*}(V)$, an edge $u v$ of $U D G(V)$ will not only be blocked by some node $w$ in $N R_{r}(u, v)$, but may also be blocked when either $\mathrm{D}_{2}$ or $\mathrm{D}_{3}$ happens. Therefore, $N G_{r}^{*}(V)$ constitutes a subgraph of $N R_{r}(V)$, which means that the maximum node degree of $N G_{r}^{*}(V)$ is no worse than its original version. In the following theorem, we show that the upper bound of $d_{\max }\left(N G_{r}(V)\right)$ in Theorem 6 remains correct in $d_{\max }\left(N G_{r}^{*}(V)\right)$ and the correctness is, for any case of $V$, not subject to Assumption $A S$.

Theorem 7. For any set $V$ of nodes on $\Re^{2}$, for all $0 \leq r \leq 1$,

$$
d_{\max }\left(N G_{r}^{*}(V)\right) \leq\left\lceil\frac{\pi}{\sin ^{-1}(r / 2)}\right\rceil .
$$

Proof. Using the same argument as Theorem 6, we assume for contradiction that two edges $u v$ and $u w$ in $N G_{r}^{*}(V)$ enclose an angle $\theta^{\prime}<2 \sin ^{-1}(r / 2)$ at node $u$. Without loss of generality, we assume that $\|u w\| \leq\|u v\|$. If $\|u w\|<\|u v\|$, the argument of Theorem 6 has proved the contradiction. Consider $\|u w\| \leq\|u v\|$ : Let $w^{\prime}$ be a point crossed by $C(u,\|u v\|)$ and the outlier of $D\left(m_{u v}, l_{u v}\right)$, as shown in Fig. 7. The two edges $w^{\prime} u$ and $u v$ enclose an angle $\theta^{\prime}$. By the law of cosines, we have

$$
\begin{aligned}
\cos \theta^{\prime} & =\frac{\left\|u w^{\prime}\right\|^{2}+(\|u v\| / 2)^{2}-\left\|m_{u v} w^{\prime}\right\|^{2}}{\left\|u w^{\prime}\right\|\|u v\|} \\
& =\frac{\|u v\|^{2}+(\|u v\| / 2)^{2}-l_{u v}^{2}}{\|u v\|\|u v\|}=1+r^{2} / 2 .
\end{aligned}
$$

Then, one corresponding right-angle triangulation is as Fig. 4c. In this case, $\sin \left(\theta^{\prime} / 2\right)=r / 2$. Thus, we can get that $\theta<\theta^{\prime}=2 \sin ^{-1}(r / 2)$. Since $\|u w\| \leq\|u v\|$, both $P(w)$ and $P(v)$ are on $C(u,\|u v\|)$. The fact that $\theta<\theta^{\prime}$ further limits $P(w)$ on the arc intersected by $D\left(m_{u v}, l_{u v}\right)$. Similarly, $P(v)$ is limited on the arc intersected by $D\left(m_{u v}, l_{u v}\right)$ for the same reason. Therefore, $P(w)$ and $P(v)$ are on the regions defined in $\mathrm{D}_{2}$, with respect to edges $u w$ and $u v$, respectively.
Next, the existence of $u v$ and $u w$ should be determined by their identifiers. If $i d(v)>i d(w), u v$ is blocked by $w$. Otherwise, if $i d(v)<i d(w)$, uw is blocked by $v$. As a sequel, no matter what the values of $i d(v)$ and $i d(w)$ are, at least one of the edges enclosing $\theta^{\prime}$ cannot be in $N G_{r}^{*}(V)$. Thus, we proved this theorem.

From Theorem 7, we can see that $d_{\max }\left(N G_{r}^{*}(V)\right)$ is constant when $r$ is sufficiently large. Therefore, there is some setting of $r$ such that $d_{\max }\left(N G_{r}^{*}(V)\right)$ is bounded by some constant for any set $V$ of $n$ nodes. So, we reach the following conclusion:

Corollary 2. The maximum node degree of the extended r-neighborhood graph is partially bounded.

In the rest, we show that $N G_{r}^{*}(V)$ inherits all desired properties achieved by $N R_{r}(V)$, except the generality for $R N G(V)$. The fact that $N G_{r}^{*}(V) \subseteq N G_{r}(V)$ confirms the planarity of $N G_{r}^{*}(V)$, since $N R_{r}(V)$ is planar for any $r$. Moreover, when $r=0$, the two arcs defined in $\mathrm{D}_{2}$ and $\mathrm{D}_{3}$ are empty. Thus, whether an edge is in $N G_{r}^{*}(V)$ is solely dependent on $\mathrm{D}_{1}$, which means that $N G_{0}^{*}(V) \equiv N G_{0}(V) \equiv G G(V)$. Therefore, $N G_{r}^{*}(V)$ remains a general structure of $G G(V)$.

However, as shown in Theorem 7 , some adjacent edges having the same length in $R N G(V)$ would be avoided in $N G_{r}^{*}(V)$. Thus, $R N G(V)$ is not always a subgraph of $N G_{r}^{*}(V)$. This means that $N G_{1}^{*}(V)$ is not essentially equivalent to $R N G(V)$. Even more, $N G_{1}^{*}(V)$ could be a subgraph of $R N G(V)$. Therefore, $N G_{r}^{*}(V)$ is no longer a general structure of $R N G(V)$.

About the connectivity, because $R N G(V)$ is not always a subgraph of $N G_{r}^{*}(V)$, we cannot ensure the connectivity of $N G_{r}^{*}(V)$ directly from that of $R N G(V)$. Therefore, we apply an entirely different logic to prove this property. The idea is based on comparing the lexicographic orders of nodes pairs. This idea has been successfully used to prove the connectivity of $X T C(V)$ [32], another subgraph of $R N G(V)$.

We define a three-field tuple $(\|u v\|, i d(u), i d(v))$ for each node pair $(u, v)$. The lexicographic order of $(u, v)$ is smaller than that of another node pair $(s, t)$ if one of the following three cases happens: 1) $\|u v\|<\|s t\|, 2)\|u v\|=\|s t\|$, 

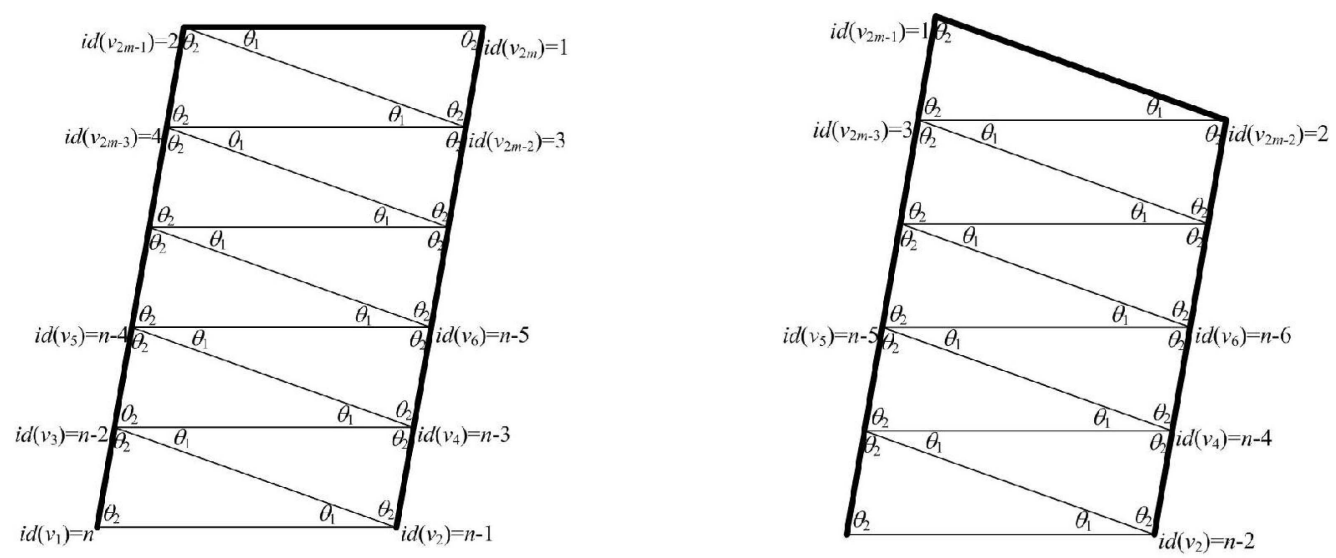

Fig. 8. A worst-case instance $V$ of $n$ nodes in $N G_{r}^{*}(V)$ : (a) $n$ is even. (b) $n$ is odd.

and $i d(u)<i d(s)$, or 3) $\|u v\|=\|s t\|, \quad i d(u)=i d(s)$ and $i d(v)<i d(t)$. Now, we prove the connectivity of $N G_{r}^{*}(V)$ in Theorem 8 .

Theorem 8. For any set $V$ of nodes on $\Re^{2}$, if the underlying $U D G(V)$ is connected, $N G_{r}^{*}(V)$ is connected, for all $0 \leq r \leq 1$.

Proof. Suppose $U D G(V)$ is connected. Let $U(V)$ be the set of unconnected nodes pairs in $N G_{r}^{*}(V)$. We assume for contradiction that some nodes pairs in $N G_{r}^{*}(V)$ are not connected, i.e., $U(V)$ is not empty. Let $(u, v)$ be the node pair with smallest lexicographic order in $U(V)$. Assume that edge $u v$ is not in $U D G(V)$, i.e., $\|u v\|>1$. Since $U D G(V)$ is connected, there must be some path longer than one hop connecting $u$ and $v$. Let $\pi(u, v)$ be such a path in $U D G(V)$. Since $\|u v\|>1$, the lengths of each edge on $\pi(u, v)$ is less than $\|u v\|$. When this path is mapped to $N G_{r}^{*}(V)$, there are some node pairs on $\pi(u, v)$ unconnected in $N G_{r}^{*}(V)$. Thus, some unconnected node pair on $\pi(u, v)$ has length shorter than $\|u v\|$, which, however, contradicts that $(u, v)$ has the smallest lexicographic order in $U(V)$. Therefore, edge $u v$ must be in $U D G(V)$.

Since edge $u v$ is in $U D G(V)$ and not in $N G_{r}^{*}(V)$, there must be some node $w$ satisfying one of the three conditions in Definition 4. Besides, either $(u, w)$ or $(w, v)$ is in $U(V)$; otherwise $(u, v)$ can be connected by path $u w v$. We consider the three cases:

1. If $\mathrm{D}_{1}$ happens, $P(w) \in N R_{r}(u, v)$. So, we have $\|u w\|<\|u v\|$ and $\|w v\|<\|u v\|$, which means that the lexicographic orders of $(u, w)$ and $(w, v)$ are less than that of $(u, v)$.

2. If $\mathrm{D}_{2}$ happens, we have $\|w v\|=\|u v\|$ and $i d(u)>i d(w)$, which means that the lexicographic order of $(w, v)$ is less than that of $(u, v)$;

3. If $\mathrm{D}_{3}$ happens, we have $\|u w\|=\|u v\|$ and $i d(v)>i d(w)$, which means that the lexicographic order of $(u, w)$ is less than that of $(u, v)$.

Therefore, we cannot find any node pair in $U(V)$ having the smallest lexicographic order. In other words, $U(V)$ is empty, which, however, is a contradiction. Thus, we have proven this algorithm.
Due to the fact that $N G_{r}^{*}(V) \subseteq N G_{r}(V)$, there may be some paths in $N G_{r}(V)$ not in $N G_{r}^{*}(V)$. Therefore, $\rho\left(N G_{r}^{*}(V)\right)$ is no better or even worse than $\rho\left(N G_{r}(V)\right)$. Even so, the upper bound of $\rho_{N G_{r}^{*}(V)}(U D G(V))$ can be as good as that proven in Theorem 5 . We briefly explain this: All arguments in Theorem 5 are not related to the two additional conditions $\mathrm{D}_{2}$ and $\mathrm{D}_{3}$, except those referred from Lemma 2. Whichever $\mathrm{D}_{1}, \mathrm{D}_{2}$, or $\mathrm{D}_{3}$ happens, $\|u w\| \leq\|u v\|$, $\|v w\|=\|u v\|$, and $\|m v\|<l$, which means that all inequalities in the proof of Lemma 2 are unchanged. Consequently, Theorem 5 is still correct, even if all conditions of Definition 4 are considered. So, $\rho\left(N G_{r}^{*}(V)\right)$ is also partially bounded.

Below, we show that the bound $1+r^{\alpha}(n-2)$ in Theorem 5 is not only correct, but also asymptotically tight to the worst possible value of $\rho_{N G_{*}^{*}(V)}(U D G(V))$. In other words, it is very hard to find another upper bound of $\rho_{N G_{r}^{*}(V)}(U D G(V))$ better than ours. We apply the same argument as that used to verify the tightness of the length stretch factor [3] and the power stretch factor [15] of $R N G(V)$

Theorem 9. For any $n \geq 2$ and $0 \leq r \leq 1$, there is a set $V$ of $n$ nodes such that

$$
\sup _{|V|=n} \rho_{N G_{r}^{*}(V)}(U D G(V))>1+r^{\alpha}(n-2)-\varepsilon,
$$

for any sufficient small $\varepsilon>0$.

Proof. Let $\theta_{1}=2 \sin ^{-1}(r / 2)-2 \lambda$ and $\theta_{2}=\pi / 2-\sin ^{-1}(r / 2)+\lambda$, where $\lambda>0$. We construct a set of $n$ nodes $V=\left\{v_{1}, v_{2}, \ldots\right.$, $\left.v_{2 m-1}, v_{2 m}, \ldots, v_{n}\right\}$, where $n \geq 2$ is even and $m=n / 2$ as follows:

1. $\left\|v_{1} v_{2}\right\| \leq 1$ and $\left\|v_{i} v_{i+1}\right\|=\left\|v_{1} v_{2}\right\|$, for

$$
i=2,3, \ldots, 2 m-1
$$

2. $\angle v_{i} v_{i+1} v_{i+2}=\theta_{1}$, for $i=1,2, \ldots, 2 m-2$;

3. $\angle v_{i+2} v_{i} v_{i+1}=\angle v_{i} v_{i+2} v_{i+1}=\theta_{2}$, for $i=1,2, \ldots, 2 m-2$;

4. $\quad i d\left(v_{i}\right)=n-i+1$, for $i=1,2, \ldots, n$.

One corresponding $U D G(V)$ is as shown in Fig. 8a. For $i=1,2, \ldots, 2 m-2$, since $\angle v_{i} v_{i+1} v_{i+2}=\theta_{1}<2 \sin ^{-1}(r / 2)$ and $\left\|v_{i} v_{i+1}\right\|=\left\|v_{i+1} v_{i+2}\right\|$, by the argument in Theorem 7 , we get $P\left(v_{i+2}\right) \in D\left(v_{i}, v_{i+1}\right) \cap C\left(m_{v_{i} v_{i+1}}, l_{v_{i} v_{i+1}}\right)$. That is, 
$P\left(v_{i+2}\right)$ is in the regions with respect to edge $v_{i} v_{i+1}$, defined in $\mathrm{D}_{2}$. Moreover, $i d\left(v_{i}\right)>i d\left(v_{i+2}\right)$. Thus, edge $v_{i} v_{i+1}$ is not in $N G_{r}^{*}(V)$. Then, the remaining edges are exactly a path (spanning tree) $v_{1} v_{3} v_{5} \ldots v_{2 m-3} v_{2 m-1} v_{2 m} v_{2 m-2} \ldots v_{6} v_{4} v_{2}$ of $V$, connecting all nodes, as the bold links in Fig. 8a. Therefore, we can get that

$$
p\left(\pi_{N G_{r}^{*}(V)}^{*}\left(v_{1}, v_{2}\right)\right)=\sum_{i=1}^{2 m-2} r^{\alpha}\left\|v_{i} v_{i+2}\right\|^{\alpha}+\left\|v_{2 m-1} v_{2 m}\right\|^{\alpha} .
$$

As $\lambda \rightarrow 0, \theta_{1} \rightarrow 2 \sin ^{-1}(r / 2)$, which implies that $\left\|v_{i} v_{i+2}\right\| \rightarrow$ $r\left\|v_{i} v_{i+2}\right\|=r\left\|v_{1} v_{2}\right\|$ according to (3). Consequently, as $\lambda \rightarrow 0$, we get that

$$
\begin{aligned}
& \sum_{i=1}^{2 m-2} r^{\alpha}\left\|v_{i} v_{i+2}\right\|^{\alpha}+\left\|v_{2 m-1} v_{2 m}\right\|^{\alpha} \\
& \rightarrow \sum_{i=2}^{2 h-2} r^{\alpha}\left\|v_{1} v_{2}\right\|^{\alpha}+\left\|v_{1} v_{2}\right\|^{\alpha} \\
& =\left\|v_{1} v_{2}\right\|^{\alpha}\left((n-2) r^{\alpha}+1\right) .
\end{aligned}
$$

On the other hand, since $\left\|v_{1} v_{2}\right\| \leq 1$, we get

$$
p\left(\pi_{U D G(V)}^{*}(u, v)\right)=\|u v\|^{\alpha} .
$$

Therefore, as $\lambda \rightarrow 0, \rho_{N G_{r}(V)}(U D G(V)) \rightarrow 1+r^{\alpha}(n-2)$. That is, $\sup _{|V|=n} \rho_{N G_{r}^{*}(V)}(U D G(V))>1+r^{\alpha}(n-2)-\varepsilon$, for any sufficient $\varepsilon>0$. For any odd $n \geq 2$, the result can be obtained by applying the same argument to the instance as shown in Fig. 8b. So, we proved this theorem.

Actually, an equivalent structure of $N G_{r}^{*}(V)$, without an original version like $N R_{r}(V)$, was mentioned in our previous paper ${ }^{3}$ [9]. In that preliminary work, however, only qualitative results were given. To prove the quantitative results, we separate $N R_{r}(V)$ from $N G_{r}^{*}(V)$ in this paper because $N R_{r}(V)$ has a clearer form in definition that can be used to highlight the main tricky parts in our derivations. Besides, all qualitative results in [9] are reevaluated here using different arguments.

\section{Purely localized Algorithm}

In this section, we propose an efficient purely localized algorithm, named PLA, to construct the $r$-neighborhood graph. This algorithm consists of two main procedures, GETINF and FINDNB. First, GETINF collects a set of nodes' information within one-hop distance, denoted as $I N_{u}$. Then, the collected information will be fed into FINDNB to determine a set of neighbors in $N R_{r}(V)$, denoted as $N B_{u}$.

\section{ALGORITHM PLA}

Input: A ratio $0 \leq r \leq 1$.

Output: A set of neighbors adjacent to $u$.

Step 1: $I N_{u}:=\operatorname{GETINF}(u, r)$;

Step 2: $N B_{u}:=\operatorname{FINDNB}\left(u, r, I N_{u}\right)$;

Step 3: Stop and output $N B_{u}$;

3. The term " $r$-neighborhood graph" in [9] does not refer to the original version in Definition 3, but to the extended version in Definition 4. In this paper, we reuse the same term to name the original version and rename the previous structure in [9] the extended version.
To collect the one-hop information, the simplest way is to let each node broadcast its information at the maximum transmission range 1 and gather the information from others. However, the severe path loss and the frequent change in topology may cause considerable power in such transmission. Therefore, in GETINF, we aim to reduce the transmission range during construction. The main idea is to incrementally raise the transmission power from a small range and then use some rule to stop the increment earlier before the transmission range 1 is reached. The detailed steps are explained as follows: The transmission range is initiated at a small distance $d_{0}$, and then it will be incrementally raised for several rounds. Let $d_{1}$ and $d_{2}$ be the previous and the current transmission ranges of a round, respectively. In each round, a node broadcasts a request to distance $d_{2}$ and waits for the responses from the receiving nodes to gather the nodes' information. To avoid replying to a node for the second time, the request of a node $u$ contains the position $P(u)$ and the previous distance $d_{1}$. As a node $v$ receives this request, it calculates the euclidean distance $\|u v\|$. Then, if $\|u v\|>d_{1}, v$ responds with its information, $P(v)$, to $u$ at distance $\|u v\|$; otherwise, it just neglects the request. In each round, the range is increased by multiplying $\sqrt[a]{2}$, which means the transmission power is multiplied by 2 each time. The process is continued until the following stopping criterion is satisfied. Let $v_{1}$ and $v_{2}$ be two crossed points intersected by $C(u,\|u v\|)$ and $C(m, l)$; see Fig. 9a. We define $S C(u, v)$ to be the semicircle enclosed by $u v_{1}$ and $u v_{2}$ with radius $\varepsilon$, where $\varepsilon>0$ is a small value less than the distance between any pair of nodes in $V$. Then, given a distance $d$, a semicircle $\chi(u, d)$ is defined as follows:

$$
\chi(u, d)=\bigcup_{\|u v\| \leq d} S C(u, v) .
$$

We can prove that, if $\chi(u, d)$ is exactly the circle $C(u, \varepsilon)$, like Fig. $9 \mathrm{~b}$, then a disk centered at $u$ with $d$ radius can cover all neighbors of $u$ in $N R_{r}(V)$. In other words, GETINF can be halted as $\chi\left(u, d_{2}\right) \equiv C(u, \varepsilon)$. Let $N_{u}(G(V))$ be the set of neighbors of node $u$ in a graph $G(V)$. This property is proven in Lemma 7.

Lemma 7. Given a node $u \in V$ and distance $d \in \Re$, if $\chi(u, d) \equiv C(u, \varepsilon)$,

$$
N_{u}\left(N G_{r}(V)\right) \subseteq\{v \in V \mid P(v) \in D(u, d)\} .
$$

Proof. We assume for contradiction that some node $s$ in $N_{u}\left(N G_{r}(V)\right)$ is not in $\{v \in V \mid P(v) \in D(u, d)\}$. Since $\varepsilon$ is less than the distance between any pair of nodes in $V$, we get $\|u s\|>\varepsilon$. Thus, edge us intersects a point on the circle $C(u, \varepsilon)$. Due to the fact that $\chi(u, d) \equiv C(u, \varepsilon)$, us must intersect at least one semicircle that composes $\chi(u, d)$; see Fig. 9b. Let $S C(u, v)$ be one of the semi-circles intersected by $u s$. Then, $u s$ is enclosed by $u v_{1}$ and $u v_{2}$ in $S C(u, v)$. In other words, $\angle s u v \leq \angle v_{1} u v$ or $\angle s u v \leq \angle v u v_{2}$. According to the argument in Theorem 7 , we can get that $\angle v_{1} u v=\angle v u v_{2}$ $=2 \sin ^{-1}(r / 2)$. Therefore, we have $\angle s u v \leq 2 \sin ^{-1}(r / 2)$. Moreover, since $s$ is not in $\{v \in V \mid P(v) \in D(u, d)\}, s$ must be farther than $v$ from $u$. So, $P(v) \in N R_{r}(u, s)$. According to Definition 4, us is not in $N R_{r}(V)$, which, however, contradicts that $s$ is a neighbor of $u$ in $N R_{r}(V)$. Thus, we conclude this lemma. 


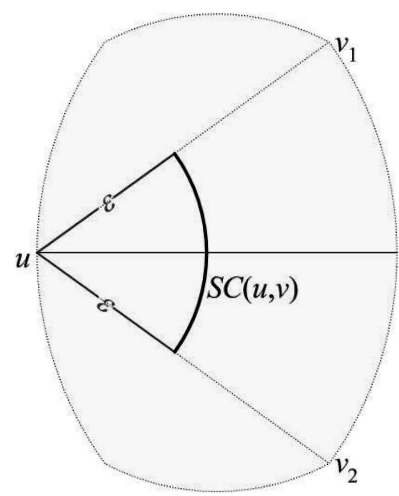

(a)

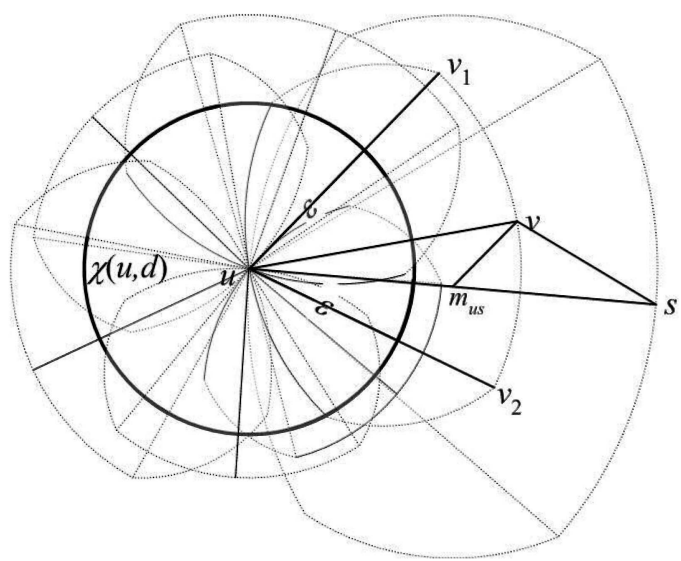

(b)

Fig. 9. (a) The semicircle $S C(u, v)$. (b) $\chi(u, d)$ is the union of all $S C(u, v)$, where $v$ is within distance $d$.

The total transmission power used by GETINF could be as large as $d_{0}^{\alpha}\left(1+2^{1}+2^{2}+\cdots+2^{I}\right)$, where $I$ is the number of rounds. This result could be worse than the maximum transmission power 1 as $I$ is large. Fortunately, when $n$ is large, nodes are closer to and evenly surrounded by each other so that $\chi(u, d)$ has more chance to be quickly shaped as $C(u, \varepsilon)$. So, we can benefit from GETINF in higher probability as the number of nodes increases.

The steps of GETINF are described below. Neglecting the communication overhead at step 2, the execution time of GETINF is dominated by the union operation at step 4 . This step can be implemented by some search-and-merge algorithm. Thus, the time complexity of GETINF is $O(n \log n)$.

\section{$\operatorname{GETINF}(u, r)$}

Step 1: $d_{1}:=0, d_{2}:=d_{0}, I N:=\phi, \chi\left(u, d_{2}\right):=\phi$;

Step 2: Broadcast a request $\left(P(u), d_{1}\right)$ to distance $d_{2}$ and gather a set $R$ of responses from nodes within $d_{1}$ and $d_{2}$;

Step 3: For each $v \in R$ do

Step 4: $I N:=I N \cup R$;

$$
\chi\left(u, d_{2}\right):=\chi\left(u, d_{2}\right) \cup S C(u, v) ;
$$

Step 5: If $d_{2} \leq 1$ and $\chi\left(u, d_{2}\right)$ is not the circle $C(u, \varepsilon)$ do

$$
\begin{aligned}
& d_{1}=d_{2} ; \\
& d_{2}:=d_{2} \times 2^{1 / \alpha} ;
\end{aligned}
$$

Return to step 2;

Step 6: Stop and output $I N$;

Now, we discuss the communication cost of GETINF. As $d_{0}$ is multiplied by $\sqrt[\alpha]{2}$ over $\alpha \log _{2}\left(1 / d_{0}\right)$ times, it is larger than 1 . Therefore, the number of rounds to increase the transmission range $d_{2}$ is dominated by $\alpha \log _{2}\left(1 / d_{0}\right)+1$. Assume a node's position can be encoded by $\log _{2} n$ bits. Each node has to broadcast at most $\left(\log _{2} n\right)\left(\alpha \log _{2}\left(1 / d_{0}\right)+1\right)$ bits for the request messages. In addition, a node will reply to the same node no more than once. Thus, a node needs at most $\left(\log _{2} n\right)(n-1)$ bits to reply to all requests. Combining these results, the communication cost of a node is no more than $\left(\log _{2} n\right)\left(\alpha \log _{2}\left(1 / d_{0}\right)+n\right)$ bits.

Once the information $I N_{u}$ is collected, node $u$ can start to determine its neighbors in $N R_{r}(V)$. One institutive way is to apply Definition 2 on $I N_{u}$ directly, as in the following procedure:

Step 1: $N:=I N_{u}$;

Step 2: For each node $v$ in $N$ do

For each node $w \in I N_{u}$ do

If $P(w) \in N R_{r}(u, v)$ do $N:=N-\{v\}$;

Step 3: Output $N$ and stop;

In this procedure, the existence of a neighbor $v$ in $I N_{u}$ is determined by checking whether some node $w$ is located in $N R_{r}(u, v)$. The correctness is obvious, while, in the worst case, it should take $O\left(n^{2}\right)$ time on each node. This time is usually not tolerable when topology changes frequently. Therefore, we aim to reduce the time complexity in this part. In FINDNB, the main idea is to reverse the original procedure. That is, instead of checking whether some node $w$ can block an edge $u v$, for each $u v$, we check whether some edge $u v$ can be blocked by a node $w$ for each $w$. The procedure is below.

This checking is begun from the farthest to the closet nodes in $I N_{u}$. So, we index all elements of $I N_{u}$ in the nondecreasing order of $\|u w\|$ in Step 2. The set $N B$ contains all candidates that could be a neighbor of $u$ during the process. As a node $w$ is given, we remove from $N B$ all failed candidates that that are already blocked by $w$. After that, $w$ is added into $N B$ to be a new candidate of $N_{u}\left(N G_{r}(V)\right)$. The process continues until all $w$ s in $I N_{u}$ are considered. Now, we prove the correctness of FINDNB.

$F I N D N B\left(u, r, I N_{u}\right)$

Step 1: $N B:=\phi$;

Step 2: Index the elements of $I N_{u}$ in nonincreasing order of $\|u w\|$

Step 3: For each node $w \in I N_{u}$ with smallest index do

For each node $v \in N B$ do

$$
\text { If } P(w) \in N R_{r}(u, v) \text { do }
$$$$
N B:=N B-\{v\} ;
$$

$N B:=N B+\{w\} ;$

Step 4: Stop and output $N B$; 
Theorem 10. For any set $V$ of nodes on $\Re^{2}, N B_{u}=N_{u}\left(N G_{r}(V)\right)$, for any $u \in V$.

Proof. We prove this by showing that, for any $v \in V, v \in$ $N B_{u}$ if and only if edge $u v$ is in $N R_{r}(V)$. Suppose an edge $u v$ is in $N R_{r}(V)$. By Definition 2, there is no $w \in$ $N_{u}(U D G(V))$ such that $P(w) \in N R_{r}(u, v)$. This implies that, once $v$ is added in $N B$, there is also no $w \in I N_{u}$ such that $v$ can be removed at Step 3. Since $v \in$ $N_{u}\left(N G_{r}(V)\right) \subseteq I N_{u}$ and each node in $I N_{u}$ can be added to $N B, v$ must be in $N B$ at least one time. So, we can get that $v$ is in the final output of $N B_{u}$. Contrarily, we suppose $u v \notin N R_{r}(V)$. Some node $w \in N_{u}(U D G(V))$ is located in $N R_{r}(u, v)$. If $v \notin I N_{u}$, the result clearly follows by Lemma 7. Otherwise, $v \in I N_{u}$. In this case, all nodes blocking $u v$ are in $I N_{u}$. Besides, every node $w$ blocking $u v$ is always considered after $v$ in GETNB. Therefore, even if $v$ can be added to $N B$, there must be a node $w \in I N_{u}$ such that $v$ can be removed from $N B$ at the successive iteration. So we get $v \notin I N_{u}$.

Lemma 7 also implies that, if $u v \in N R_{r}(V)$, then $v \in N_{u}$ and $u \in N_{v}$ and that, if $u v \in N R_{r}(V)$, then $v \notin N_{u}$ and $u \notin N_{v}$. So, the neighbors (links) determined by GETNB are symmetric.

\section{Corollary 3. Any topology resulted by PLA is symmetric.}

Consider the time complexity of FINDNB. Step 2 can be done by some sorting algorithm in $O(n \log n)$. Before a node $w \in I N_{u}$ is added to $N B$, any $v \in I N$ blocked by $w$ is removed from $N B$. Therefore, for any two nodes in $N B$, neither of them can be blocked by the other. Let $s$ and $t$ be two nodes in $N B$. The argument of Theorem 7 indicates that, if $\angle s u t<2 \sin ^{-1}(r / 2)$, then either $s$ blocks $t$ or $t$ block $s$. Since neither $s$ blocks $t$ nor $t$ blocks $s$, we get that $\angle$ sut $\geq 2 \sin ^{-1}(r / 2)$. Therefore, during the process, the size of $N B$ can be never greater than $d_{\max }\left(N G_{r}(V)\right)$. Consequently, FINDNB can be done in $\mathrm{O}\left(n \max \left\{\log n, d_{\max }\left(N G_{r}\right)\right\}\right)$ time. We can observe that this time complexity depends on the parameter $r$. When $r$ is equal or close to 0 (the worst cases), the time complexity of FINDNB is still $\mathrm{O}\left(n^{2}\right)$. However, when $r$ is sufficiently large, such that $d_{\max }\left(N G_{r}(V)\right)$ is a constant, FINDNB can be done in $\mathrm{O}(n \log n)$.

With a slight modification, PLA can be easily applied on the extended $r$-neighbors graph and all results can be preserved. We omit the detailed explanation here.

\section{Conclusion}

In this paper, we proposed a purely localized structure to control the topology in wireless networks. We showed that the worst case of the power stretch factor is an increasing function of $r$ and the worst cast of the maximum node degree is contrarily a decreasing function of $r$. So, the two objectives can be adjusted in our structure. Although the

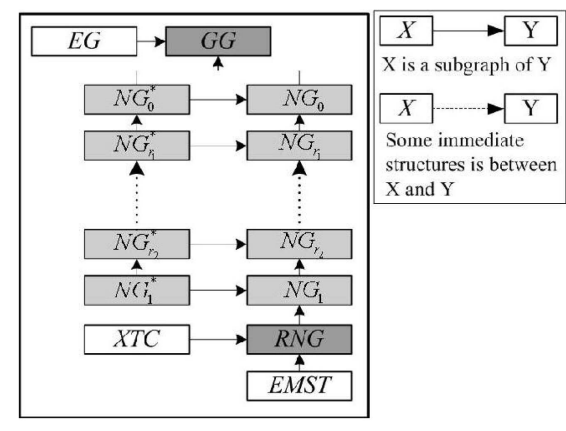

Fig. 10. The relationships of $N G_{r}(V), N G_{r}^{*}(V), G G(V)$, and $R N G(V)$.

power stretch factor is related to $n$ so that our structure is not really a spanner, $\rho\left(N G_{r}(V)\right)$ can still be bounded for some range of $r$. Therefore, the power stretch is partially bounded in our structure. About the maximum node degree, we proposed an upper bound derived for $d_{\max }\left(N G_{r}(V)\right)$. However, this result is correct only when no node has two or more neighbors at exactly the same distance. For this reason, an extended structure $N G_{r}^{*}(V)$ was given to comprehend this theorem.

Besides, the proposed structure can always result in a connected topology with symmetric edges. Any resulting topology is always planar. The relations between the $r$-neighborhood graph and existent structures are summarized in Fig. 10. Specially, $N \operatorname{Rr}(V)$ is a general structure of both $G G(V)$ and $R N G(V)$.

To construct our structure, we proposed a 1-hop purely localized algorithm, PLA. It can avoid long-distance transmission when collecting information and can be efficiently done in $\mathrm{O}(n \log n)$ time when $d_{\max }\left(N G_{r}(V)\right)$ is constant.

For further research, a localized topology control approach enables the design of localized routing protocols. For instance, the greedy route discovery in CFG [26] and GPSR [11] are based on $G G$. We anticipate that the $r$-neighborhood graph could provide a concrete basis for many interesting extensions due to the sound theoretical results. Moreover, the parameter $r$ can be turned to find the best settings for different scenarios. Another interesting issue for possible further work is to evaluate the stability of the proposed structure when perfect position (range) information is not available or when the accuracy of position information differs from node to node.

\section{APPENDIX}

Proof of Lemma 2. Without loss of generality, we assume that $\|u w\| \leq\|v w\|$. Let $y$ be the projection of $w$ on $u v$ so that $y w$ is perpendicular to $u v$. We can derive that

$$
\begin{aligned}
\|y m\| & =\frac{\|v w\|^{2}}{2\|m v\|}-\frac{\|w m\|^{2}}{2\|m v\|}-\frac{\|m v\|}{2} \text { and }\|y x\| \\
& =\sqrt{\|w v\|^{2}-\left(\frac{\|v w\|^{2}}{2\|m v\|}-\frac{\|w m\|^{2}}{2\|m v\|}+\frac{\|m v\|}{2}\right)^{2}} .
\end{aligned}
$$


Thus,

$$
\begin{aligned}
\|u w\|^{2}= & \|w y\|^{2}+(\|u m\|-\|y m\|)^{2} \\
= & \|w y\|^{2}+(\|m v\|-\|y m\|)^{2} \\
= & \|v w\|^{2}-\left(\frac{\|w v\|^{2}}{2\|m v\|}-\frac{\|w m\|^{2}}{2\|m v\|}+\frac{\|m v\|}{2}\right)^{2} \\
& +\left(\frac{3\|m v\|}{2}-\frac{\|v w\|^{2}}{2\|m v\|}+\frac{\|w m\|^{2}}{2\|m v\|}\right)^{2} \\
= & 2\|m v\|^{2}-\|v w\|^{2}+2\|w m\|^{2} .
\end{aligned}
$$

Then, the power consumed by path $u w v$ is as follows:

$$
\begin{aligned}
p(u w v) & =\|u w\|^{\alpha}+\|v w\|^{\alpha} \\
& =\left(2\|m v\|^{2}-\|v w\|^{2}+2\|w m\|^{2}\right)^{\frac{\alpha}{2}}+\|v w\|^{\alpha} .
\end{aligned}
$$

From (1), we get

$$
\|w m\|<l=\|u v\| \sqrt{1+2 r^{2}} / 2=\|m v\| \sqrt{1+2 r^{2}}
$$

and $\|v w\|<\|u v\|$, so

$$
\begin{aligned}
& \left(2\|m v\|^{2}-\|v w\|^{2}+2\|w m\|^{2}\right)^{\frac{\alpha}{2}}+\|v w\|^{\alpha} \\
& \leq\left(\left(4+4 r^{2}\right)\|m v\|^{2}-\|v w\|^{2}\right)^{\frac{\alpha}{2}}+\|v w\|^{\alpha} \\
& \leq\left(\frac{\left(4+4 r^{2}\right)}{4}\|u v\|^{2}-\|u v\|^{2}\right)^{\frac{\alpha}{2}}+\|u v\| \\
& =\left(r^{2}\|u v\|^{2}\right)^{\frac{\alpha}{2}}+\|u v\|^{\alpha}=\|u v\|^{\alpha}\left(1+r^{\alpha}\right) .
\end{aligned}
$$

Thus, we have that $p(u w v) \leq\|u v\|^{\alpha}\left(1+r^{\alpha}\right)$.

\section{ACKNOWLEDGMENTS}

This paper was supported in part by the National Science Council of the ROC, under grants NSC 95-2219-E-009-008 and NSC-95-2752-E-009-005-PAE, and in part by the New Generation Broadband Wireless Communication Technologies and Applications Project of the Institute for Information Industry, ROC.

\section{References}

[1] P. Bose, J. Gudmundsson, and P. Morin, "Ordered Theta Graphs," Proc. Canadian Conf. Computational Geometry, 2002.

[2] P. Bose, P. Morin, I. Stojmenović, and J. Urrutia, "Routing with Guarantee Delivery in Ad Hoc Networks," ACM/Kluwer Wireless Networks, vol. 7, no. 6, pp. 609-616, 2001.

[3] P. Bose, L. Devroye, W. Evans, and D. Kirkpatrick, "On the Spanning Ratio of Gabriel Graphs and Beta-Skeletons," SIAM J. Discrete Math., 2004.

[4] I. Chlamtac, M. Conti, and J.N. Liu, "Mobile Ad Hoc Networking: Imperatives and Challenges," Ad Hoc Networks, vol. 1, pp. 13-64, 2003.

[5] L. Feeney, "An Energy-Consumption Model for Performance Analysis of Routing Protocols for Mobile Ad Hoc Networks," ACM J. Mobile Networks and Applications, vol. 3, no. 6, pp. 239-249, 2001.

[6] K.R. Gabriel and R.R. Sokal, "A New Statistical Approach to Geographic Variation Analysis," Systematic Zoology, vol. 18, pp. 259-278, 1969.

[7] H.N. Gabow, J.L. Bently, and R.E. Tarjan, "Scaling and Related Techniques for Geometry Problems," Proc. ACM Symp. Theory of Computing, pp. 135-143, 1984.
[8] J. Geo, L.J. Guibas, J. Hershburger, L. Zhang, and A. Zhu, "Geometric Spanner for Routing in Mobile Networks," Proc. ACM MobiHoc, 2001.

[9] A.A.K. Jeng and R.H. Jan, "An Adjustable Structure for Topology Control in Wireless Ad Hoc Network," Proc. 2005 Int'l Conf. Wireless Network Comm. and Mobile Computing, 2005.

[10] J. Katajainen, "The Region Approach for Computing Relative Neighborhood Graphs in the Lp Metric," Computing, vol. 40, pp. $147-161,1988$

[11] B. Karp and H.T. Kung, "GPSR: Greedy Perimeter Stateless Routing for Wireless Networks," Proc. MobiCom, 2000.

[12] P. Klein, S. Rao, M. Rauch, and S. Subramanian, "Faster ShortestPath Algorithms for Planar Graph," Proc. Symp. Theory of Computing, 1994.

[13] L.A. Latiff and N. Fisal, "Routing Protocols in Wireless Mobile Ad Hoc Networks-A Review," Comm., vol. 2, pp. 600-604, 2003.

[14] W.H. Lee and T.H. Meng, "A Lower Power GPS Receiver Architecture," Proc. Global Telecomm. Conf., vol. 11, pp. 153-157, 1999.

[15] X.Y. Li, P.J. Wan, and Y. Wang, "Power Efficient and Sparse Spanner for Wireless Ad Hoc Networks," Proc. IEEE Int'l Conf. Computer Comm. and Networks, pp. 564-567, 2001.

[16] X.Y. Li and P.J. Wan, "Constructing Minimum Energy Mobile Wireless Networks," ACM SIGMOBILE Mobile Computing and Comm. Rev., vol. 5, pp. 55-67, 2001.

[17] X.Y. Li, P.J. Wan, Y. Wang, and O. Frieder, "Sparse Power Efficient Topology for Wireless Networks," Proc. 35th Ann. Hawaii Int'l Conf. System Sciences (HICSS '02), vol. 9, 2002.

[18] X.Y. Li, G. Calinescu, P.J. Wan, and Y. Wang, "Localized Delaunay Triangulation with Application in Ad Hoc Wireless Networks," IEEE Trans. Parallel and Distributed Systems, vol. 14, no. 10, pp. 1035-1047, Oct. 2003.

[19] X.Y. Li and Y. Wang, "Efficient Construction of Low Weight Bounded Degree Planar Spanner," Int'l J. Computational Geometry and Applications, vol. 14, nos. 1-2, pp. 69-84, 2004.

[20] L. Li and J.Y. Halpern, "Minimum-Energy Mobile Wireless Networks Revisited," Proc. IEEE Int'l Conf. Comm. (ICC '01), 2001.

[21] R. Prakash, "Unidirectional Links Prove Costly in Wireless AdHoc Networks," Proc. Third Int'l Workshop Discrete Algorithms and Methods for Mobile Computing and Comm. (DIAL-M), 1999.

[22] F.P. Preparata and M.I. Shamos, Computational Geometry-An Introduction. Springer-Verlag, 1985.

[23] R. Rajaraman, "Topology Control and Routing and Ad Hoc Network: A Survey," ACM SIGACT, vol. 33, no. 2, 2002.

[24] V. Rodoplu and T.H. Meng, "Minimum Energy Mobile Wireless Networks," IEEE J. Selected Areas in Comm., vol. 17, no. 8, pp. 13331344, Aug. 1999.

[25] W.Z. Song, Y. Wang, and X.Y. Li, "Energy Efficiency: Localized Algorithms for Energy Efficient Topology in Wireless Ad Hoc Networks," Proc. Fifth ACM Int'l Symp. Mobile Ad Hoc Networking and Computing, 2004.

[26] I. Stojmenovic and S. Datta, "Power and Cost Aware Localized Routing with Guaranteed Delivery in Wireless Networks," Wireless Comm. and Mobile Computing, vol. 4, no. 2, pp. 175-188, 2004.

[27] I. Stojmenovic and X. Lin, "Power-Aware Localized Routing in Wireless Networks," IEEE Trans. Parallel and Distributed Systems, vol. 12, no. 11, pp. 1122-1133, 2001.

[28] G.T. Toussaint, "The Relative Neighborhood Graph of a Finite Planar Set," Pattern Recognition, vol. 12, no. 4, pp. 261-268, 1980.

[29] Y. Wang and X.Y. Li, "Distributed Spanner with Bounded Degree for Wireless Ad Hoc Networks," Int'l J. Foundations of Computer Science, vol. 14, pp. 183-200, 2003.

[30] W. Wang, X.Y. Li, K. Moaveninejad, Y. Wang, and W.Z. Song, "The Spanning Ratios of Beta-Skeleton," Proc. Canadian Conf. Computational Geometry (CCCG), 2003.

[31] Y. Wang and X.Y. Li, "Localized Construction of Bounded Degree Planar Spanner for Wireless Ad Hoc Networks," Proc. 2003 Joint Workshop Foundations of Mobile Computing, pp. 59-68, 2003.

[32] R. Wattenhofer and A. Zollinger, "XTC: A Practical Topology Control for Ad-Hoc Networks," Proc. 18th Parallel and Distributed Processing Symp., pp. 26-30, 2004.

[33] A.C.C. Yao, "On Constructing Minimum Spanning Trees in kDimensional Spaces and Related Problems," SIAM J. Computing, vol. 11, pp. 721-736, 1982. 


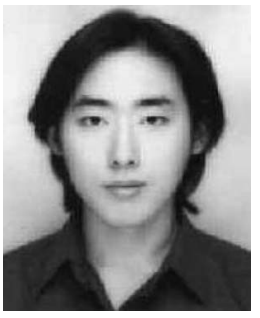

Andy An-Kai Jeng received the BS degree in statistics from Tamkang University and the MS degree in management information systems from National Chi Nan University in 2001 and 2003 , respectively. He is currently pursuing the $\mathrm{PhD}$ degree in the Department of Computer and Information Science at National Chiao-Tung University, Taiwan, Republic of China. His research interests include wireless networks, algorithm design and analysis, scheduling theory, and operations research.

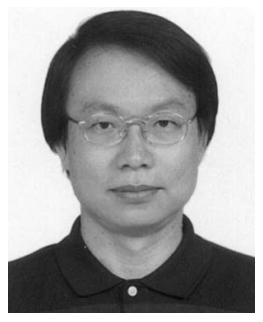

Rong-Hong Jan received the BS and MS degrees in industrial engineering and the $\mathrm{PhD}$ degree in computer science from National Tsing Hua University, Taiwan, in 1979, 1983, and 1987, respectively. He joined the Department of Computer and Information Science, National Chiao Tung University, in 1987, where he is currently a professor. From 1991-1992, he was a visiting associate professor in the Department of Computer Science, University of Maryland, College Park, Maryland. His research interests include wireless networks, mobile computing, distributed systems, network reliability, and operations research. He is a member of the IEEE.

$\triangleright$ For more information on this or any other computing topic, please visit our Digital Library at www.computer.org/publications/dlib. 